\title{
Les traces des traces de la Première Guerre mondiale : la représentation photographique des lieux de conflit et ses enjeux mémoriels
}

\section{Emmanuel Béhague}

Université de Strasbourg

\section{Abstract}

Dieser Beitrag widmet sich der fotografischen Darstellung von Kriegsschauplätzen des Ersten Weltkriegs und untersucht dabei die erinnerungspolitische Bedeutung ästhetischer Entscheidungen. Als fotografische Spur der physischen „Spuren“, die die Konflikte im Raum hinterlassen haben, ist die fotografische Repräsentation insofern zu hinterfragen, als ihr eine kollektive Bedeutung innewohnt: Einerseits streben Bilder danach, den öffentlichen Raum zu besetzen, andererseits verfügen sie über eine eigene Performativität, die auf die Wirklichkeit einwirkt. Nachdem zunächst der Begriff der Spur als Schnittstelle zwischen der Fotografie und ihrem Objekt untersucht werden soll, werden wir uns mit der kollektiven Bedeutung fotografischer Repräsentation auseinandersetzen. Dazu beschäftigen wir uns mit den Arbeiten von Michael St Maur Sheil, Guillaume Amat und Paola de Pietri, drei Fotografen und Fotografinnen, die durch die Abbildung von Konfliktschauplätzen die Notwendigkeit, sich gegen das Vergessen zu stemmen, zum Ausdruck bringen.

Consacrée à la photographie des lieux dans lesquels se sont déroulés les combats de la Première Guerre mondiale, cette contribution se penche sur la signification mémorielle des choix esthétiques. Traces photographiques des traces physiques laissées par les conflits dans l'espace, la représentation photographique doit être interrogée, dans la mesure où elle revêt une signification collective : aspirant à investir l'espace public d'une part, les images disposent d'autre part d'une performativité qui leur est propre et influent de ce fait sur le réel. Après nous être arrêtés sur la notion de trace comme point de rencontre entre la photographie et son objet, puis sur la signification collective de la représentation photographique, nous nous intéresserons à titre d'exemple au travail de Michael St Maur Sheil, Guillaume Amat et Paola de Pietri, trois photographes cherchant à exprimer à travers la photographie des lieux du conflit la nécessité d'une résistance à l'oubli.

Devoted to the photography of the places where the fighting of the First World War took place, this contribution examines the memorial significance of the aesthetic choices. Photographic traces of the physical traces left by conflicts in space, photographic representation must be questioned insofar as it has a collective meaning: aspiring to take over the public space, the images also have a performativity of their own and thus influence reality. After examining the notion of trace as a meeting point between photography and its object, and then the collective dimension of photographic representation, we will look at the work of Michael St Maur Sheil, Guillaume Amat and Paola de Pietri, three photographers who seek to express the need for resistance to oblivion through the photography of conflict sites.

\section{Keywords}

Fotografie $\bullet$ kollektive Erinnerung $\bullet$ Schlachtfelder $\bullet$ Erster Weltkrieg $\bullet$ fotografische Spur $\bullet$ Visual History

Photographie $\bullet$ mémoire collective $\bullet$ champs de bataille $•$ Première Guerre mondiale $\bullet$ trace photographique $\bullet$ histoire visuelle

Photography $\bullet$ Collective memory $\bullet$ Battlefields $\bullet$ First World War $\bullet$ Traces $\bullet$ Visual history 


\section{INTRODUCTION}

En dépit de l'élargissement considérable qu'a permis l'avènement de l'histoire visuelle dans la prise en compte des images en général et de leur statut comme objet d'étude ${ }^{1}$, la photographie en particulier demeure aujourd'hui très majoritairement appréhendée comme un document témoignant visuellement du passé. Dans une telle perspective, les clichés " d'époque » (photographies privées, photographies de propagande, cartes postales, photographies de voyage, photographie publicitaire...) permettent ainsi la reconstitution par l'historien d'une situation, d'un événement, d'une réalité sociale, politique, économique, culturelle. Si une telle entreprise contribue ainsi à la perpétuation de la mémoire de ce passé à travers une meilleure connaissance de celui-ci et de la façon dont il a été vécu, c'est tout autant le cas pour une autre approche, qui se préoccupe elle de la façon dont il est, dans l'a posteriori des événements eux-mêmes cette fois, photographiquement rendu compte de ce qu'il reste de ce passé. Une démarche photographique d'ordre artistique cette fois, c'est-à-dire empreinte de subjectivité, peut dès lors constituer un objet d'étude pour qui tente de discerner la forme que prend et la place qu'occupe l'art comme média dans le processus mémoriel. C'est dans ce second type d'approche de la photographie que s'inscrit cette contribution, consacrée à la représentation photographique des champs de bataille de la Première Guerre mondiale, c'est-à-dire des espaces profondément marqués par les événements passés. Après nous être arrêtés sur la notion de trace comme point de rencontre entre la photographie et son objet, puis sur les enjeux de la représentation photographique dans la mémoire collective, il s'agira, à travers trois études de cas, de s'intéresser à la représentation contemporaine de ces marques physiques du passé dans le présent, c'est-à-dire de faire converger questionnements esthétiques et mémoriels.

\section{UNE « TRACE DES TRACES »: DE L'ANALOGIE ENTRE LA PHOTOGRAPHIE ET LE CHAMP DE BATAILLE}

Stigmates, cicatrices, traces, blessures, meurtrissures, séquelles : nombreux sont les termes qui peuvent désigner la façon dont les combats de la Première Guerre mondiale ont posé leur empreinte sur les lieux de leur déroulement. Celui de « trace " en particulier permet, à travers sa polysémie, de rendre compte de la double nature de ces marques. La trace du passé s'inscrit dans l'espace tout d'abord comme un résidu,

Une définition que donne Gerhard Paul de l'histoire visuelle témoigne de manière synthétique de cet élargissement : " Für alle jene Versuche, die unterschiedlichen Bildgattungen als Quellen und als eigenständige Gegenstände in die historiografische Forschun einzubeziehen, Bilder sowohl als Abbildungen als auch als Bildakte zu behandeln, die V sualität von Geschichte wie die Historizität des Visuellen zu thematisieren und zu präsentieren, möchte ich den Sammelbegriff „Visual History“ vorschlagen. [...] Letztlich geht es darum, Bilder über ihre zeichenhafte Abbildhaftigkeit hinaus als Medien zu untersuchen, die Sehweisen konditionieren, Wahrnehmungsmuster prägen, historische Deutungsweisen transportieren [...]. Visual History ist somit mehr als die Geschichte der visuellen Medien; sie umfasst das ganze Feld der visuellen Praxis der Selbstdarstellung, der Inszenierung und Aneignung der Welt sowie schließlich die visuelle Medialität von Erfahrung und Geschichte » (Paul $2006: 25)$. un reste, un « laissé là » invisible parfois, dans le cas des sols empoisonnés, visible comme ces restes d'obus, ces ruines de casemate recouvertes peu à peu par la mousse et envahies par la végétation. Mais la trace y est aussi « empreinte », comme une " trace de pas ", c'est-à-dire une présence en creux, la façon dont une réalité désormais absente a modelé le paysage : ce sont les tranchées, les fossés, les béances laissées dans le sol par ces mêmes obus.

Or cette double trace caractérise précisément le processus même de la photographie, comme le rappelle Philippe Dubois :

Le point de départ, c'est donc la nature technique du procédé photographique, le principe élémentaire de l'empreinte lumineuse régie par les lois de la physique et de la chimie [...]. Cela signifie que la photographie s'apparente à cette catégorie de 'signes' où l'on trouve aussi la fumée (indice d'un feu), l'ombre portée (indice d'une présence), la cicatrice (marque d'une blessure), la ruine (vestige de ce qui a été là), le symptôme (d'une maladie), l'empreinte de pas etc. (Dubois $1990: 46$ )

" Cicatrice », « ruine », " vestige », " empreinte » : les mots utilisés par Dubois pour désigner le rapport de la photographie à son objet en général sont les mêmes que ceux qui caractérisent l'objet particulier que constituent les champs de bataille dans leur rapport aux événements du passé. La photographie, dans le cas présent, est donc bien une « trace des traces " du conflit, et crée de ce point de vue une forme de continuum non plus simplement entre le passé et le présent, comme dans le rapport qui s'instaure au moment où je regarde une photographie des soldats au combat par exemple, mais entre trois strates temporelles distinctes : le passé, les événements dont il est question ; le moment où la trace de ceux-ci - une tranchée, les vestiges rouillés d'une pièce d'artillerie - est photographiée ; et le moment où l'observateur regarde la photographie de ces traces. La photographie des champs de bataille active donc la dynamique d'un souvenir non seulement que l'observateur du présent n'a pas vécu, mais qu'il ne voit pas, ou dont il ne voit que des traces.

\section{PHOTOGRAPHIE, HISTOIRE, MÉMOIRE}

En représentant les vestiges du conflit, la photographie réinscrit dans le présent de celui qui observe la photographie d'un passé. Mais à la différence d'autres modes de représentation (filmiques, romanesques, documentaires...) ce passé n'est en réalité pas le passé représenté. L'objet de la représentation photographique se distingue de l'enjeu de celle-ci, à savoir un passé invisible, révolu, qui n'est lui-même présent que par les marques qu'il a laissées dans l'espace. Celles-ci prennent la forme d'une marque physique de l'événement lui-même, tandis que leur représentation, c'est-à-dire la façon dont elles sont rendues visibles, est elle l'expression d'une intentionnalité, celle du photographe. Or cette même représentation crée une nouvelle trace. 
Dans La mémoire, l'Histoire, L'oubli, Paul Ricœur, s'attachant au sens de l'empreinte comme forme particulière de la trace, distingue trois emplois de ce dernier terme : la trace écrite, objet d'étude de l'historien, " l'empreinte corporelle, cérébrale, corticale " (Ricœur 2000 : 17) objet des neurosciences, et l'« impression en tant qu'affection résultant du choc d'un événement dont on peut dire qu'il est frappant, marquant » (ibid. : 16). La réception de l'image photographique, c'est-à-dire l'impression qu'elle produit chez son observateur, paraît au premier abord étrangère à ces trois formes. Néanmoins, cette image déclenche potentiellement des émotions, des pensées, des souvenirs, des réflexions. Au prix d'un élargissement de la définition de Ricœur, mais qui n'en trahit pas pour autant la signification, on peut donc considérer que la photographie comme restitution visuelle de la marque physique du paysage produit ce qui constituerait un cas particulier de la trace affective, particulier en ce que celle-ci résulte non de l'événement passé lui-même, mais de la représentation de ce qu'il en reste. C'est donc précisément parce qu'elle affecte, à travers la réception, le présent qu'il convient de s'intéresser à la forme de cette représentation, de cette trace des traces.

Car cette réception dans laquelle l'affect a part est aussi collective : si l'image photographique est l'expression d'une subjectivité, de la « personnalité » du photographe, la démarche artistique n'est pourtant personnelle que dans un certain sens. En effet, les photographies, si elles sont prises, le sont pour être vues, pour circuler, elles aspirent à investir l'espace public. Dans le cas de la photographie des champs de bataille, ceci est d'autant plus important que cet investissement peut être porté par des institutions publiques. Comme on le verra, un des trois projets évoqués ici a bénéficié, sinon du label à proprement parler de la " Mission Centenaire », groupe d'intérêt public mis en place en 2012 par le gouvernement français, du moins de son soutien, qui se traduit en particulier par la mise en ligne des photographies sur le site officiel de la Mission. L'institution mémorielle mise en place par le pouvoir politique valide donc une démarche artistique personnelle, lui conférant ainsi une autorité particulière.

La question de cette autorité revêt d'autant plus d'importance lorsqu'on s'attache aux effets que produisent les photographies. Dressant un tableau des avancées d'une histoire visuelle qu'il cherche à légitimer comme discipline à part entière, Gerhard Paul distingue trois façons d'aborder les images : les images comme source («Bilder als Quellen »), les images comme médias (« Bilder als Medien »), les images comme forces génératrices («Bilder als generative Kräfte ») (Paul 2014). Pour l'historien, qui s'appuie notamment sur les théories d'Horst Bredekamp, les images ne sauraient désormais plus être appréciées en fonction de leur seule valeur informationnelle, mais en ce qu'elles produisent ce qu'elles montrent, et contribuent ainsi à façonner l'histoire : «Bilder geben Bredekamp zufolge Geschichte nicht nur passivisch wieder, sondern vermögen sie aufgrund der besonderen « Triebkraft der Form » wie jede Handlung oder Handlungsanweisung auch zu prägen » (Paul 2014).
Ce faisant, elles contribuent, tout autant qu'elles « représentent » l'histoire, à en produire une interprétation et à la véhiculer, et jouent de ce fait un rôle essentiel dans les dynamiques de construction et de stabilisation des identités collectives. Enfin, elles sont elles-mêmes déclencheuses d'effets, c'est-à-dire qu'elles sont en mesure de générer de nouvelles réalités politiques, culturelles, sociales. Pour Bredekamp, les images constituent des " actes", leur modus operandi relève d'un " toucher " qui modifie la constitution de celui qui les regarde. On peut alors parler d'un " acte d'image » (« Bildakt ») défini de la manière suivante : « [Der Bildakt ist] eine Wirkung auf das Empfinden, Denken und Handeln [...], die aus der Kraft des Bildes und der Wechselwirkung mit dem betrachtenden, berührenden und auch hörenden Gegenüber entsteht » (Bredekamp 2013 : 52). Qu'elles soient documentaires ou artistiques, figées ou en mouvement, les images sont porteuses d'une performativité, d'une puissance intrinsèque :

Rapportée à son essence, l'image n'est que représentation, objet second ; mais déterminée par sa puissance, elle peut être plus que son référent, comme un paysage photographié par Sophie Ristelhueber qui nous émeut plus que le même paysage vu de nos propres yeux. Étudier l'image quant à sa puissance, c'est aussi observer qu'un film sur les camps de concentration produit d'autres effets qu'une simple description des camps. (Dierkens, Golsenne 2010 : 25)

On comprend alors où réside la spécificité de la photographie, dans laquelle cette puissance intrinsèque se double de ce qui la distingue d'autres images (telles que l'œuvre picturale ou la caricature), à savoir son apparente analogie avec le réel. L'image photographique puise en effet la force particulière qui est la sienne dans son ontologie même, le fameux " ça-a-été » de Roland Barthes (Barthes 1980). L'image photographique, elle-même impression (photo-graphie, c'est-à-dire écriture par la lumière) laisse donc une empreinte en ce qu'elle modifie le réel. Lorsqu'elle se donne pour objet le passé, à travers la trace physique des conflits, cela signifie qu'elle influe potentiellement sur la mémoire. À ce titre, sa production marquée de subjectivité engage le photographe, dont la démarche, quoi que personnelle, a toujours en soi une signification collective. Et lorsque le pouvoir politique s'en saisit, celle-ci entre dans le domaine des usages politiques du passé.

\section{MICHAEL ST MAUR SHEIL, FIELDS OF BATTLE - LANDS OF PEACE 1914-1918}

Les photographies de Michael St Maur Sheil illustrent parfaitement ce dernier cas de figure ${ }^{2}$. En effet, son travail sur les

2 Pour les trois photographes évoqués ici, nous appuierons notre commentaire sur les photographies disponibles en ligne, dans la mesure où l'objet de cette contribution n'est en effet pas de livrer une étude exhaustive des travaux considérés (ce qui aurait impliqué un travail en liver une étude exhaustive des travaux considérés (ce qui aurait impliqué (archive), mais de considérer ceux-ci comme des propositions artistiques immédiatement accessibles, c'est-à-dire visibles dans l'espace public. 
champs de bataille du premier conflit mondial, intitulé Fields of battle - Lands of peace 1914-1918, apparaît sur le site de la «Mission centenaire " ${ }^{3}$, et a été exposé du 2 avril au 3 août 2014 sur les grilles du Jardin du Luxembourg à Paris, avant de circuler en France et à travers le monde. Cette validation institutionnelle de la démarche artistique ainsi que cette présence dans un lieu à la fois fréquenté et chargé d'histoire viennent s'ajouter à une forte présence sur internet. Outre les différents sites sur lesquels est évoqué ou commenté le projet, deux ont été conçus par le photographe lui-même. Le premier rassemble divers documents en rapport avec le projet et à des manifestations autour de celui-ci, ainsi que des liens directs vers des médias ayant commenté le travail ${ }^{4}$. Le second apporte d'autres informations sur cette série ainsi que sur des réalisations apparentées du photographe, et documente le déroulement du travail entrepris en incluant au passage des recommandations hôtelières dans les régions traversées et l'invitation lancée aux visiteurs du site internet à prendre contact pour participer à des parcours guidés par l'artiste lui-même ${ }^{5}$.

Si cette présence médiale du photographe britannique mériterait sans doute un exposé à elle seule, on s'arrêtera ici sur le projet photographique en lui-même. Si l'on met entre parenthèses le genre du portrait ou la photographie d'objet, dans lesquels la mise en scène du sujet (dans le sens photographique du terme) est explicite et essentielle, l'acte photographique entretient au réel comme objet de représentation un rapport ambigu. Dans la mesure où c'est bien la lumière qui "écrit » (et non le photographe) là où c'est le peintre qui peint, s'opère une délégation partielle dans la réalisation de l'image qui contredit le principe même d'un agencement intentionnel du réel : le hasard, en photographie, a sa place, il est le co-auteur de l'œuvre photographique, même si celle-ci est une re-présentation dont le photographe définit les modalités. Celui-ci dispose de moyens technologiques qui lui permettent de donner une forme à la saisie photographique de ce réel. À la différence des deux autres démarches artistiques présentées ici, que caractérise une ligne formelle stricte, Michael St Maur Sheil a développé un projet diversifié, dans lequel se côtoient différents sujets photographiques tels que les restes des combats sous la forme de structures architecturales ou d'objets, des vues aériennes, des photographies de lieux mémoriels.

Deux principes esthétiques de cette grammaire visuelle, complémentaires, sont néanmoins récurrents. Le travail du photographe se caractérise tout d'abord par une recherche poussée d'effets divers de luminosité et de couleurs. Ainsi, la crête de Messines (Belgique), théâtre de batailles en 1914 et 1917, sert de cadre à la représentation d'un coucher de

https://centenaire.org/fr/autour-de-la-grande-guerre/photographie-artistique/fields-battle-terres-de-paix-14-18. Dernière consultation le 15 juillet 2021.

4 https://www.fieldsofbattle1418.org/photographer.html. Dernière consultation le 18 juillet 2021

5 http://www.westernfrontphotography.com/. Dernière consultation le 18 juillet 2021 C'est à ces différents sites que nous empruntons les exemples commentés. II faut enfi évoquer l'existence d'un site internet concerné à la commercialisation des produits dérivès de l'exposition, permettant de commander des mugs, posters, puzzles représentant telle ou telle photographie, ainsi que des coussins de tailles diverses... permettant ainsi littéralement de s'endormir sur le souvenir de la Première Guerre mondiale (https://fieldsofbattle. printstoreonline.com/, dernière consultation le 18 juillet 2021). soleil sur fond gris ; une photographie aérienne de la Somme représentant les tranchées et les cratères formés par les explosions se caractérise avant tout par l'unicité et la luminosité de la couleur verte qui se dégage de l'image ; le blanc immaculé de la neige et celui du ciel se fondent dans le cimetière de Tyne Cot, à Ypres. Par ailleurs, nappes de brume et brouillard apparaissent à plusieurs reprises dans les photographies, qu'ils s'élèvent de marais en forêt à Authuille (Somme) ou recouvrent la totalité d'un vaste paysage au soleil couchant à Villers-Bretonneux (Somme). Cette manière de souligner les couleurs et leur combinaison, ces jeux avec l'opacité du brouillard confèrent donc aux situations photographiées une aura particulière qui détermine la réception par-delà ce qui est représenté, à savoir les traces elles-mêmes. Si « belles » soient-elles, les photographies se caractérisent dès lors par une forme de déréalisation, une caractéristique qui peut paraître problématique au regard de la raison d'être initiale du projet, qui est de contribuer à perpétuer le souvenir du conflit. À ce travail sur la lumière s'ajoute un autre procédé esthétique qui consiste à mettre en évidence un objet précis en le plaçant au premier plan : un vieux ballon de football, une montre à gousset, des obus rongés par la corrosion, les restes d'un canon. Par cette mise en scène, le regard de l'observateur est dirigé sur l'objet comme protagoniste principal, l'espace, c'est-à-dire le lieu du conflit faisant alors office de décor. Cette focalisation extrême produit une forme de surcharge signifiante, dans laquelle la trace résiduelle ne se contente plus d'être un indice renvoyant au passé, mais se voit érigée en symbole.

Ramener ces caractéristiques esthétiques à la question de la trace conduit ainsi à un double constat. Celle-ci, dans de nombreuses photographies, semble mise à profit pour la création d'une image photographique qui recherche davantage l'efficacité visuelle, par-delà (ou plutôt que) l'exploration de la question de la mémoire. Plus qu'à une réflexion sur ce qu'il reste du conflit dans le présent, le spectateur semble invité à une simple contemplation de l'image. L'autre trait esthétique récurrent fonctionne de manière inverse : la trace indicielle, sous la forme du résidu, est mise au premier plan, une expression à comprendre tant dans son sens littéral que figuré. La photographie semble dès lors traduire une forme d'injonction au souvenir.

\section{GUILLAUME AMAT, ESPACES MÉMORIELS}

Dans ses Espaces mémoriels ${ }^{6}$, le photographe Guillaume Amat pratique le montage de deux clichés, procédé qui constitue le principe de la série. Le premier, réalisé par le photographe lui-même, représente un lieu du combat aujourd'hui : un champ, une colline enneigée, une zone herbeuse parsemée des béances laissées par les explosions, le tracé d'une tranchée. Le second est une photographie prise à l'époque sur plaque de verre : des soldats dans une tranchée souvent, une explosion, un attroupement devant une 
chapelle entre-temps disparue, des cadavres de chevaux Par-delà le contraste entre la couleur et le noir et blanc présent sur la nouvelle photographie ainsi conçue, la scène du passé semble le plus souvent se fondre parfaitement dans l'environnement. Dans quelques cas, l'incohérence temporelle s'exprime visuellement à travers la présence, dans le cadre de la photographie, de rares promeneurs venus parcourir les anciens champs de bataille.

Le photographe procède ainsi à une forme d' " actualisation » des images d'archives ${ }^{7}$ dont il convient de saisir les enjeux mémoriels en revenant au « noème » de la photographie selon Roland Barthes, le «ça-a-été » :

J'appelle référent photographique non pas la chose facultativement réelle à quoi renvoie une image ou un signe mais la chose nécessairement réelle qui a été placée devant l'objectif, faute de quoi, il n'y aurait pas de photographie. La peinture, elle, peut feindre la réalité sans l'avoir vue [...]. Au contraire, dans la photographie, je ne peux jamais nier que la chose a été là. II y a double position conjointe : de réalité et de passé. Et puisque cette contrainte n'existe que pour elle, on doit la tenir, par réduction, pour l'essence même, le noème de la photographie sera donc : 'Ça-a-été' [...]. (Barthes 1980 : 120)

C'est donc d'un dépassement du " ça-a-été » barthien que relève la démarche de Guillaume Amat. Ces réalisations constituent autant de fictions photographiques, dans le but de lutter contre l'oubli :

Quand on se déplace dans ces sites, Arras, Verdun etc., c'est assez étrange, on ne sait plus très bien, on a le sentiment d'être dans un lieu de mémoire et parfois dans un parc d'attraction. Un panneau indique les tranchées, des paysages vides et bosselés qui ressemblent à des terrains de golf. II est difficile de percevoir quoi que ce soit de la guerre dans ces espaces reconstitués ou laissés pratiquement intacts comme des territoires mémoriels. Alors on cherche ce qu'on ne pourra pas trouver : les soldats, les hommes et leurs histoires. D'où cette idée de juxtaposer ces morceaux de paysages, lieux de mémoires avec des vues historiques ${ }^{8}$.

Le procédé mis en œuvre vient donc remettre en cause les trois strates temporelles évoquées plus haut, et crée l'illusion d'un présent qui n'a pas existé : la photographie ne constitue dès lors plus une trace. De ce point de vue, ce qu'écrit le photographe lui-même à propos du projet est révélateur :

Dans un premier temps j'ai acheté sur internet des vues stéréoscopiques sur plaque de verre en choisissant celles qui montraient des scènes du quotidien [...]. Quelques légendes étaient visibles sur ces documents d'histoire et je me suis efforcé de retrouver dans la mesure du possible les lieux de

7 Selon les termes du photographe lui-même : "Mélangées aux images actuelles, les images d'archives en noir et blanc très réelles se fondent dans une image couleur qu les actualisent. " Ibid.

8 https://www.guillaumeamat.com/personal-projects/espaces-memoriels. Dernière consultation le 18 juillet 2021. prise de vue. Puis au fur et à mesure, il m'est apparu sans importance de chercher une telle précision. Car la guerre a eu lieu à l'échelle de territoires et de régions. La guerre a pris place sur les collines, les plaines, les prairies, les forêts, les rivières, les arbres, la boue, le sable. Partout où les hommes sont tombés ${ }^{9}$.

La démarche ainsi décrite est donc révélatrice d'un basculement du geste de l'historien vers celui de l'artiste. II ne s'agit plus d'ancrer la démarche artistique dans une forme de factualité en faisant converger topographiquement le lien entre le passé et le présent, mais de développer un propos photographique consacré à la mémoire collective. En procédant à la fusion du présent et du passé, l'artiste produit une illusion esthétique dans laquelle disparaît le souvenir comme acte, comme dynamique. Son travail s'oppose en cela à celui de la photographe Paola de Pietri.

\section{PAOLA DE PIETRI, TO FACE}

La série To face de la photographe italienne Paola de Pietri, qui a donné lieu à des expositions à Rome, Milan, Epinal, Turin et Toulouse, rassemble des photographies prises entre 2008 et 2011 le long du tracé de l'ancien front autrichien et italien, des Alpes au plateau du Karst (Carso). Dans une topographie tout à fait différente de celle de l'Ouest, car presque exclusivement montagneuse, s'est formée la ligne de front italo-autrichienne, avec ses tranchées creusées à même le roc, ses cavernes, ses postes d'artillerie. La série suit une ligne esthétique rigoureuse, caractérisée par une grande sobriété, la reprise de quelques éléments matériels (de l'herbe, de la pierre, de la neige), dans des paysages montagneux vides de toute présence humaine. Originaire de la province de Reggio d'Émilie, c'est à travers les récits de ses parents, qui les tenaient de ses grands-parents qu'elle a eu connaissance des événements qui se sont déroulés dans les montagnes. C'est dans cette dynamique de transmission qu'elle situe sa propre démarche, concevant ainsi l'acte photographique comme un moyen prendre le relais de la transmission orale : " Historical facts have survived in the form of direct personal accounts that are now approaching the edge of thier existence. I have tried to pick up the last surviving remnants of this thin thread of memory in the very places where events unfurled $[\ldots]^{10}$. "

À l'instar de St Maur Sheil, la raison d'être de la démarche artistique est donc celle de la préservation de la mémoire par l'utilisation de la saisie photographique comme trace du réel. D'un point de vue esthétique néanmoins, tout semble opposer les deux artistes. Ainsi, De Pietri privilégie un prisme limité de couleurs, toujours directement associées à la matérialité de l'objet (le gris de la pierre, le blanc de la neige, le vert de l'herbe), rendant ainsi compte de la rigueur du climat et des conditions vécues par les combattants un siècle plus tôt. Par ailleurs, la distance temporelle ne fait pas l'objet d'une mise

\footnotetext{
9 Ibid.

10 https://www.paoladepietri.com/to-face/. Dernière consultation le 15 juillet 2021.
} 
en scène à travers l'utilisation d'une trace résiduelle placée en premier plan. Ici au contraire, les cicatrices du paysage, terme employé par la photographe, sont le plus souvent décentrées dans le paysage, et de taille réduite. Elles ne sont pas invisibles, mais ne se manifestent au regard que dans un second temps et s'apparentent au punctum, élément de la photographie qui, prenant souvent la forme d'un détail, vient interrompre le studium. Si le punctum, en soi, peut recouvrir toute sorte de réalité ${ }^{11}$, on ne peut que constater la parenté frappante entre les termes employés par le philosophe pour le définir et la notion de trace elle-même :

Un mot existe en latin pour désigner cette blessure, cette piqûre, cette marque faite par un instrument pointu ; ce mot m'irait d'autant mieux qu'il renvoie aussi à l'idée de ponctuation et que les photos dont je parle sont en effet comme ponctuées, parfois même mouchetées, de ces points sensibles ; précisément, ces marques, ces blessures sont des points. Ce second élément qui vient déranger le studium, je l'appellerai donc punctum ; car punctum, c'est aussi : piqûre, petit trou, petite tache, petite coupure - et aussi coup de dés. Le punctum d'une photo, c'est ce hasard qui, en elle, me point (mais aussi me meurtrit, me poigne $)^{12}$

Comme chez St Maur Sheil enfin, le brouillard est souvent présent chez la photographe italienne, sous une forme radicalement différente toutefois. Loin de ne constituer qu'un arrière-plan ou de contribuer à créer une atmosphère, il constitue davantage un mouvement dans lequel s'estompent

11 Quelques exemples fournis par Barthes sont des souliers à brides sur un cliché de James van der Zee, les dents d'un enfant dans un quartier de New-York photographié par William Klein, les ongles d'Andy Warhol dans un portrait de Duane Michals (Barthes 1980 73-77).

12 On objectera que le punctum relève davantage d'une présence fortuite du détail, ce qui ne saurait être le cas pour le travail de De Pietri, précisément consacré aux traces. Une distinction évidente entre ce qui relèverait du hasard de la scène photographiée d'une part et de l'intentionnalité présumée du photographe d'autre part est cependant toujours difficile, comme en témoigne le premier exemple fourni par le philosophe, à savoir un cliché pris par Koen Wessing au Nicaragua en 1979. les formes de la montagne, devenant ainsi une métaphore de l'oubli progressif. Par-delà les traces elles-mêmes, ces photographies disent tout autant la dynamique de leur lente car naturelle - disparition :

The photographs record the gradual disintegration of the scars left on the mountain by warfare. They show how the nature is absorbing this trenches, caves, peaks ravaged by mines, thousands of bomb craters and the ruins of sheds and storerooms constructed with local raw materials ${ }^{13}$.

\section{CONCLUSION}

Le caractère spectaculaire des photographies de Michael St Maur Sheil, la sobriété de Paola de Pietri et les montages atemporels de Guillaume Amat constituent autant de propos photographiques dans lesquels les options esthétiques sont portées par une réflexion sur la mémoire du premier conflit mondial. Par-delà des différences évidentes, un même souci est sensible de contrecarrer la dynamique de l'oubli. Si on ne peut apprécier la réalité de la trace " affective » effectivement laissée par ces représentations, dans la mesure où celle-ci relève d'une réception individuelle, la signification collective de telles démarches est manifeste dès lors qu'on a présent à l'esprit ce qui constitue la raison d'être des images en général, qui est d'être visibles. Cette même signification de la photographie comme cas particulier de ces images est de ce point de vue d'autant plus évidente que celle-ci, « quelle qu'elle soit, semble entretenir avec la réalité visible une relation plus innocente, et donc plus exacte, que les autres objets mimétiques » (Sontag 2008 : 19). Son intérêt en est d'autant plus important comme objet d'étude pour l'historien de la mémoire.

13 https://www.paoladepietri.com/to-face/. Dernière consultation le 15 juillet 2021.

\section{Bibliographie}

Barthes, Roland (1980), La chambre claire. Note sur la photographie, Paris, Gallimard/Seuil.

Bredekamp, Horst (2013), Theorie des Bildakts, Frankfurt am Main, Suhrkamp.

Dierkens, Alain / Golsenne, Thomas (2010), « Une théorie des actes d'image ", in Bartholeyns, Gil / Dierkens, Alain / Golsenne, Thomas (éd.), La Performance des images, Bruxelles, Publications de I)Université de Bruxelles, 15-25.

Dubois, Philippe (1990), L'acte photographique, Paris, Nathan.

Paul, Gerhard (2006), « Von der historischen Bildkunde zur Visual History. Eine Einführung », in Paul (éd.), Visual History. Ein Studienbuch, Göttingen, Vandenhoeck \& Ruprecht.
Paul, Gerhard (2014), "Visual History », Version : 3.0, in: Docupedia-Zeitgeschichte, Zentrum für zeithistorische Forschung Potsdam, http://docupedia.de/zg/paul_visual_history_v3_de_2014, dernière consultation le 19 juillet 2021.

Ricœur, Paul (2000) La mémoire, l'histoire, l'oubli, Paris, Editions du Seuil, 2000

Serres, Alexandre (2002), "Quelle(s) problématique(s) de la trace ? ", HAL : sic_00001397, https://archivesic.ccsd.cnrs.fr/ sic_00001397, dernière consultation le 19 juillet 2021.

Sontag, Susan, (2008 [1979]), « Dans la caverne de Platon », in Sur la photographie, Paris, Christian Bourgois. 